\title{
GENERIC DIFFERENTIABILITY OF ORDER-BOUNDED CONVEX OPERATORS
}

\author{
JONATHAN M. BORWEIN ${ }^{1}$
}

(Received 28 March 1985; revised 14 October 1985)

\begin{abstract}
We give sufficient conditions for order-bounded convex operators to be generically differentiable (Gâteaux or Fréchet). When the range space is a countably order-complete Banach lattice, these conditions are also necessary. In particular, every order-bounded convex operator from an Asplund space into such a lattice is generically Fréchet differentiable, if and only if the lattice has weakly-compact order intervals, if and only if the lattice has strongly-exposed order intervals. Applications are given which indicate how such results relate to optimization theory.
\end{abstract}

\section{Introduction}

Convex analysis plays a central role in the study of optimality conditions and in non-linear analysis. Vector-valued convex operators occur naturally in a variety of settings. This was illustrated in [1], [2] and we give further examples in Section 4 below. There has also been considerable interest in the differentiability properties of non-linear operators, both for theoretical and applied reasons. If derivatives are known to exist sufficiently often (almost everywhere or on a dense $G_{\delta}$ subset) then one can often reduce the problem being studied to a more tractable differentiable problem. Moreover, convex operators are the most accessible class of non-linear operators, and as such demand study even if one is more directly interested in other, say Lipschitz, operators.

In our previous papers [1], [2] we studied the existence of subgradients for continuous convex operators, and gave various results on the generic differentiability of continuous convex operators. Kirov [4], [5] has continued this study,

\footnotetext{
${ }^{1}$ Dalhousie University, Canada

(C) Copyright Australian Mathematical Society 1986, Serial-fee code 0334-2700/86
} 
primarily by the use of generalized monotone operators. In [5] he observes that much more can be said if the operators are required to be order-bounded rather than merely convex. In this paper, we adapt the techniques of [1] and [3] to establish differentiability results for order-bounded convex operators between ordered Banach spaces. We also show that when the range space is an order-complete Banach lattice, our conditions are both necessary and sufficient. These results considerably extend various theorems given in [5].

We commence by recalling necessary facts and notations. The reader is directed to [1] and [7] for further details. For simplicity we restrict ourselves to Banach space. Let $X$ be a Banach space and let $Y$ be a (partially) ordered Banach space with closed normal positive cone $S$. We denote the induced order by $\leqslant$ or $\leqslant s$. (Recall that $S$ is normal if and only if there is an equivalent renorming with $0 \leqslant s_{s} y \leqslant{ }_{s} x$ implying $\|y\| \leqslant\|x\|$.) As elsewhere we adjoin an abstract " $\infty$ " to $Y$ and $S$ and consider mappings $f$ between $X$ and $Y \cup\{\infty\}$, written $\dot{Y}$. Then $f: X \rightarrow \dot{Y}$ is $\left(S\right.$-) convex if for $0 \leqslant t \leqslant 1$ and $x_{1}, x_{2}$ in $\operatorname{dom} f:=\{x \in X: f(x) \in$ $Y$ \} one has

$$
f\left(t x_{1}+(1-t) x_{2}\right) \leqslant s t f\left(x_{1}\right)+(1-t) f\left(x_{2}\right) .
$$

We will say that $f$ is order-bounded at $\bar{x}$ in $\operatorname{dom} f$ if one can find a neighbourhood $N$ of zero and some $y \in Y$ such that

$$
\bar{x}+N \subset\left\{x \in X: f(x) \leqslant{ }_{s} y\right\} .
$$

Obviously such an $\bar{x}$ lies in $\operatorname{int}(\operatorname{dom} f)$. Moreover, when $f$ is convex and order-bounded at some $\bar{x}$, it is actually order-bounded throughout $\operatorname{int}(\operatorname{dom} f)$. We will call such a mapping (locally) order-bounded. Since the cone is normal, order-bounded convex maps are continuous; but the converse obtains only when $\operatorname{int}(S)$ is non-empty. In general, even such nice convex mappings as the absolute value on a Banach lattice are not order-bounded.

Let us also recall that the cone $S$ is Daniell if every positive decreasing net converges. When $Y$ is a Banach lattice this is equivalent to the norm being order-continuous, [7, Theorem 5.11]. We make one new definition. We will say that an order interval $[0, x]:=\left\{y \in Y: 0 \leqslant s_{s} y \leqslant x\right\}$ is strongly exposed (by $\phi$ in $[0, x]^{+}:=\left\{g \in Y^{*}: g(y) \geqslant 0\right.$ for $\left.\left.y \in[0, x]\right\}\right)$ if, for all $\varepsilon>0$ there exists $\delta>0$ such that

$$
0 \leqslant s_{s} y \leqslant{ }_{s} x \text { and } \phi(y) \leqslant \delta \text { implies }\|y\|<\varepsilon .
$$

If we may only assert that

$$
0 \leqslant{ }_{s} y \leqslant s \text { and } \phi(y)=0 \text { implies } y=0
$$

we say that the interval is exposed (by $\phi$ ).

Finally, a Banach space $X$ is an Asplund space, respectively a weak Asplund space, if every extended real-valued convex function on $X$ is generically Fréchet, respectively Gâteaux, differentiable throughout the interior of its domain. (A set 
is generic if it contains a dense $G_{\delta}$.) Asplund spaces include reflexive spaces and separable dual spaces; weak Asplund spaces include all weakly compact generated spaces and so all separable spaces. (See [1] and references therein.)

\section{Sufficient conditions for generic differentiablity}

Our central result is:

THEOREM 2.1. Let $X$ be a Banach space, let $Y$ be an ordered Banach space whose cone $S$ is closed and normal, and let $f: X \rightarrow \dot{Y}$ be order-bounded and $S$-convex. Suppose $S$ is Daniell.

a] If $X$ is an Asplund space and order intervals in $Y$ are strongly exposed, then $f$ is generically Fréchet differentiable throughout the interior of its domain.

b] If $X$ is a weak Asplund space and order intervals in $Y$ are exposed, then $f$ is generically Gâteaux differentiable throughout the interior of its domain.

Proof. Let $\bar{x}$ in $\operatorname{int}(\operatorname{dom} f)$ be given. Select $y$ in $Y$ and a ball $N$ around zero such that (1.2) holds.

Let $x \in \bar{x}+N$. Then, as $f$ is convex,

$$
y-f(\bar{x}) \geqslant f(\bar{x}+x)-f(\bar{x}) \geqslant f(\bar{x})-f(\bar{x}-x) \geqslant f(\bar{x})-y,
$$

and $f(\bar{x}+N)$ lies in an order interval, $[a, b]$. Again by convexity, for $x$ in $\bar{x}+\frac{1}{2} N$ and $h$ in $\frac{1}{2} N$ we have

$$
f(x)-f(x-h) \leqslant \frac{f(x+t h)-f(x)}{t} \leqslant \frac{f(x+s h)-f(x)}{s}
$$

for $0<t \leqslant s \leqslant 1$. Since $f(x)-f(x-h) \geqslant a-b$, and as $s$ is Daniell, the directional minorant

$$
\nabla f(x ; h):=\inf _{t>0} \frac{f(x+t h)-f(x)}{t}
$$

exists for $x$ in $\bar{x}+\frac{1}{2} N$ and $h$ in $X$. Moreover, $\nabla f(x ; \cdot)$ is convex and finite and, again since $S$ is Daniell,

$$
\nabla f(x ; h)=\lim _{t \downarrow 0} \frac{f(x+t h)-f(x)}{t} .
$$

a] Now, let $\phi$ strongly expose $[0, b-a]$. Since $f$ is $S$-convex with $f(\bar{x}+N) \subset$ $[a, b]$ while $\phi \in[0, b-a]^{+}, \phi f$ is convex on $\bar{x}+N$. Since $X$ is Asplund, there is a dense $G_{\delta}$ subset, $G$, in $\bar{x}+N$ such that $\phi f$ is Fréchet differentiable at points of $G$. We show (much as in [1]) that $f$ is actually Fréchet differentiable on $G$. Let $x$ lie in $G$. First observe that, for $0<t<1$

$$
0 \leqslant \frac{f(x+t h)-f(x)}{t}-\nabla f(x ; h) \leqslant 2(b-a),
$$


for $x \in \bar{x}+\frac{1}{2} N$ and $h \in \frac{1}{2} N$. Also $\nabla \phi f(x ; h)=\phi \nabla f(x ; h)$ and, as $\nabla \phi f(x ; \cdot)$ is linear, we have

and

$$
0 \leqslant \nabla f(x ; h)+\nabla f(x ;-h) \leqslant 2(b-a)
$$

$$
\phi(\nabla f(x ; h)+\nabla f(x ;-h))=0 .
$$

Since $\phi$ exposes $[0, b-a],(2.3)$ and (2.4) show that $\nabla f(x ; \cdot)$ is linear, being both sublinear and homogeneous. This, in conjunction with (2.1), shows that $f$ is linearly Gâteaux differentiable at $x$. To complete the argument let $\varepsilon>0$ be given and choose $\delta>0$ to satisfy (1.3) with $x:=2(b-a)$. Then, as $\phi f$ is Fréchet at $x$, we may find $\gamma>0$ so that when $h$ lies in $\frac{1}{2} N$

$$
\frac{\phi f(x+t h)-\phi f(x)}{t}-\nabla \phi f(x ; h) \leqslant \delta
$$

for $0<t<\gamma$. Since (2.2) holds, we have

$$
\left\|\frac{f(x+t h)-f(x)}{t}-\nabla f(x ; h)\right\| \leqslant \varepsilon
$$

if $0<t<\gamma$ and $h \in \frac{1}{2} N$. As $\nabla f(x ; \cdot)$ is linear and continuous we are done.

b] This follows as in the first part of the previous proof.

Conditions for a cone to be Daniell were discussed in detail in [1]. Conditions for exposed intervals are as follows:

Proposition 2.1. Let $Y$ be a Banach space partially ordered by a normal closed cone $S$.

a] Order intervals in $Y$ are exposed if

(i) $S$ has separable order intervals; or (ii) $S$ has a base; or (iii) $Y$ has an equivalent strictly convex renorm which is S-monotone $(0 \leqslant y \leqslant x$ implies $\|y\| \leqslant$ $\|x\|)$.

b] Order intervals in $Y$ are strongly exposed if

(i) $S$ has norm compact intervals; or (ii) $S$ has a bounded base; or

(iii) $Y$ has equivalent locally uniformly convex renorm which is $S$-monotone.

Proof. Let $x$ in $S$ be fixed with $x \neq 0$.

a] (i) The cone generated by the order interval $[0, x]$ is separable and so has a base, $B,[1]$ and as the space is locally convex we may separate 0 and $B$ to produce an exposing functional. This also establishes (ii). In case (iii) we argue that the unique tangent, $\phi$, to the renormed strictly convex ball $N:=\{y \in Y$ : $\|y\| \leqslant\|x\|\}$ exposes $x$ in $N$ and, by monotonicity, exposes $x$ in $[0, x]$. But then $\phi$ exposes $[0, x]$ as well.

b] (i) Since $[0, x]$ is exposed by a] (i) and compact (every sequence has a convergent subsequence) it is strongly exposed; indeed, otherwise we have $\varepsilon>0$ and $\phi\left(x_{n}\right)$ tending to 0 for $\left\|x_{n}\right\| \geqslant \varepsilon$ and $0 \leqslant x_{n} \leqslant x$. Since $\left(x_{n}\right)$ has a convergent 
subsequence in norm, this is impossible. (ii) was established in [1]. (iii) Now $\phi$ strongly exposes the renormed locally uniformly convex ball at $x$ and so strongly exposed 0 in $[0, x]$.

If the domain is not Asplund or weakly Asplund, or if the operator is not order-bounded, the examples given in [1] show that Theorem 2.1 will generally fail.

We continue by studying the case in which $Y$ is a lattice.

\section{Lattice characterizations}

We suppose now that $Y$ is a Banach lattice (a complete normed vector lattice whose norm satisfies $\|y\| \leqslant\|x\|$ whenever $|y| \leqslant|x|)$. The key result is:

Proposition 3.1. Let Y be a Banach lattice. Then the following are equivalent:

i] $Y$ has a lattice equivalent locally uniformly convex Banach lattice renorming.

ii] Order intervals in $Y$ are strongly exposed.

iii] Order intervals in $Y$ are weakly compact.

iv] The lattice cone in $Y$ is Daniell.

Proof. i] $\Rightarrow$ ii]. Since strong exposure is preserved by lattice isomorphisms, this follows from b] (iii) of Proposition 2.1. ii] $\Rightarrow$ iii]. If $Y$ possesses a non-weakly compact order interval then one can construct a lattice orthogonal norm one sequence $\left(x_{n}\right)$ in $Y$ with $0 \leqslant x_{n} \leqslant x_{0}$ for all $n,[7$, p. 94]. Now

$$
s_{n}:=\sum_{k=1}^{n} x_{k}=\bigvee_{k=1}^{n} x_{k} \leqslant x_{0} \text { since } x_{k} \wedge x_{J}=0 \text { for } k \neq j
$$

Hence, for any positive $\phi$ in $Y^{*},\left(\phi\left(s_{n}\right)\right)$ is isotone and majorized. Thus $\phi\left(x_{n}\right)$ tends to zero. Since $Y$ is a Banach lattice $\left(x_{n}\right)$ is weakly convergent to 0 . This certainly means that $\left[0, x_{0}\right]$ is not strongly exposed, as each $x_{n}$ is norm one. iii] $\Rightarrow$ iv]. This implication holds for any partial order [1]. iv] $\Rightarrow$ i]. Since $Y$ is a Daniell Banach lattice, $Y$ is order continuous and we apply the Davis-Ghoussoub-Lindenstrauss renorming theorem [3] to complete the hard step. (The theorem guarantees a locally uniformly convex lattice equivalent renorm for an order continuous Banach lattice.)

As observed in [6, p. 28], it is also equivalent to assume that $Y$ has a lattice-equivalent Kadec norm. Note also that every $\sigma$-finite $L_{\infty}(\mu, E)$ has a lattice-equivalent strictly-convex lattice renorming. Simply let $E:=\bigcup_{n=1}^{\infty} E_{n}$ where $\mu\left(E_{n}\right) \leqslant 1$, and let $\|\cdot\|$ be given by $\|f\|:=\|f\|_{\infty}+\sum_{n=1}^{\infty} 2^{-n}\left\|f \mid E_{n}\right\|_{2}$. 
Also, in $L_{p}(\mu), 1 \leqslant p<\infty$, (with the standard ordering), it is easy to exhibit the strongly exposing functional for $[0, \bar{x}]$. We have $\phi:=\bar{x}^{p-1} \in L_{q}(\mu)(q+p=$ $p q)$ and $0 \leqslant y \leqslant \bar{x}$ implies $\phi(y)=\int \bar{x}^{p-1} y d \mu \geqslant\|y\|^{p}$.

THEOREM 3.1. Let $Y$ be a countably order-complete Banach lattice. Then the following are equivalent.

i] Order intervals in $Y$ are strongly exposed.

ii) Order intervals in $Y$ are weakly compact.

iii] Suppose that $f: X \rightarrow \dot{Y}$ is convex and order-bounded while $X$ is an Asplund space. Then $f$ is generically Fréchet differentiable.

iv] Suppose that $f: X \rightarrow \dot{Y}$ is convex and order-bounded while $X$ is a weak Asplund space. Then $f$ is generically Gâteaux differentiable.

v] Suppose that $f: \mathbf{R} \rightarrow Y$ is convex and order-bounded. Then $f$ is generically Gâteaux differentiable.

vi] $Y$ contains no Banach sub-lattice isomorphic to $l_{\infty}(\mathbf{N})$.

PROof. i] $\Leftrightarrow$ ii] follows from Proposition 3.1. ii] $\Leftrightarrow$ iii]. Since the cone is normal and Daniell, Theorem 2.1 a] now applies. ii] $\Leftrightarrow$ iv] follows similarly from part b] of the theorem. Clearly iii] implies v] and iv] implies v]. To complete the circle we establish that v] implies vi] and vi] implies ii]. v] $\Rightarrow$ vi]. Suppose that $Y$ contains a lattice copy of $1_{\infty}(\mathbf{N})$. There is no loss in assuming $Y=l_{\infty}(\mathbf{N})$. Then let $\left\{r_{n}\right.$ : $n \in \mathbf{N}\}$ be chosen dense in $[-1,1]$. Let $f: \mathbf{R} \rightarrow l_{\infty}(\mathbf{N})$ be defined (as in [4]) by

$$
f(r):=\sup _{n \in \mathbb{N}}\left|r-r_{n}\right| .
$$

Clearly, $f$ is convex and order-bounded. Moreover, if $|r|<1, f$ is not Gâteaux differentiable at $r$. Indeed, since $\left\{r_{n}: n \in \mathbf{N}\right\}$ is dense in $[-1,1]$ we may calculate that

$$
\limsup _{\varepsilon \rightarrow 0^{+}} \frac{f(r+\varepsilon)+f(r-\varepsilon)-2 f(r)}{\varepsilon}=2 \text {, }
$$

and so $f$ is nowhere Gâteaux differentiable on $(-1,1)$. (Note that, nonetheless, $f$ has a unique linear subgradient whenever $r \notin\left\{r_{n}: n \in \mathbf{N}\right\}$.)

vi] $\Rightarrow$ ii]. Since $Y$ is countably order-complete this follows from [7, Theorem 5.14].

The equivalences fail if $Y$ is not countably order-complete. Indeed, $f(x):=|x|$ on $X:=Y:=C[0,1]$ is nowhere Gâteaux differentiable on $N:=\{x \in X: \| x-$ $\left.\bar{x} \|<\frac{1}{2}\right\}$ where $\bar{x}(t):=1-2 t$ for $0 \leqslant t \leqslant 1$, [1]. This is not entirely obvious, but follows after some routine but tedious calculations. 
Kirov's Corollaries in [5] regarding Fréchet differentiability or order-bounded convex operators (established by entirely different methods) are all special cases of Theorem 3.1, sometimes with redundant hypotheses. He requires $X$ to be a reflexive Banach space and $Y$ to be a Banach lattice such that either a) intervals are norm compact, or b) intervals in $Y$ and $Y^{*}$ are weakly compact, or c) intervals in $Y$ are weakly compact and $f$ has only compact subgradients.

\section{Applications}

a] We consider the following vector convex program (VCP):

$$
h(u):=\inf _{s} f(x) \text { subject to } g(x) \leqslant{ }_{k} u \text {. }
$$

We assume that $f: X \rightarrow \dot{Y}$ is $S$-convex and that $g: X \rightarrow \dot{U}$ is $K$-convex. We suppose that int $K$ is non-empty and that Slater's condition holds: there exists $\hat{x}$ in $\operatorname{dom} f$ with $g(\hat{x}) \in-$ int $K$. We also suppose that $(Y, S)$ is a Banach lattice with weakly compact order intervals, and so is order-complete.

Then, as in [1], [2], $h$ defines another $S$-convex mapping; which is actually locally order-bounded as a consequence of Slater's condition. (More general constraint qualifications ensure continuity but not order-boundedness.) Thus, if we assume that $h(0)$ is finite, $h$ is order-bounded and convex on a neighbourhood of zero. In particular, Theorems 2.1 and 3.1 apply to $h$ and give conditions for $h$ to be generically differentiable. As explained in [1], if $h$ is differentiable at $u$ with Gâteaux derivative $T$, then $-T$ is the unique Lagrange multiplier for (VCP). In fact, if $h$ is Fréchet differentiable at $u$ we may conclude that the subgradient of $h$ is norm-to-norm upper semi-continuous at $u,[1]$.

b] Suppose now that $f:=A$ and $g:=B$ are continuous linear mappings. Then (VCP) becomes a form of the abstract Farkas lemma. Such inequality systems are central to the study of positive operators [7].

As outlined in a] the differentiability points of $h(u):=\inf _{s}\left\{A x \mid B x \leqslant{ }_{k} u\right\}$ correspond to unique Lagrange multipliers. In this case $T=\nabla h(u)$ if and only if $T$ is the unique linear operator solution to

$$
T v \leqslant{ }_{s} h(v), \quad \forall v \in U
$$

and

$$
T u=h(u) .
$$

This in turn means that $T$ is the unique solution in $L(U, Y)$ to

$$
T B=A, T(K) \subset-S, T u=h(u) .
$$


c] Let $f: X \times T \in \mathbf{R}$ be convex in $x \in X$ and measurable in $t \in T$. Suppose that $X$ is an Asplund space and that one can find $k \in L_{p}(T)(1 \leqslant p<\infty)$ such that

$$
f(x, t) \leqslant k(t)
$$

if $\left\|x-x_{0}\right\|<\varepsilon$, for some $\varepsilon>0, x_{0} \in X$. We define a convex operator $F$ : $X \rightarrow L_{p}(T)$ by $F(x)(t):=f(x, t)$. Then (4.5) guarantees that $F$ is locally orderbounded. Theorem 3.1 applies and we may conclude that generically $F$ is Fréchet differentiable.

\section{Acknowledgement}

This research was supported by NSERC operating and travel grants, and completed while the author was visiting Cambridge University's Management Science Division.

\section{References}

[1] J. M. Borwein, "Continuity and differentiability properties of convex operators", Proc. London Math. Soc. 3 (1982), 420-444.

[2] J. M. Borwein, "Subgradients of convex operators", Math. Operationsforch. Statist. Ser. Optim. 15 (1984), 179-191.

[3] W. J. Davis, N. Ghossoub, and J. Lindenstrauss," "A lattice renorming theorem and applications to vector-valued processes", Trans. Amer. Math. Soc. 90 (1981), 531-540.

[4] N. K. Kirov, "Differentiability of convex mappings and generalized monotone mappings", $C$. R. Acad. Bulgare Sci. 34 (1981), 1.

[5] N. K. Kirov, "Generalized Fréchet differentiability of convex operators", Proc. Amer. Math. Soc. 94 (1985), 97-102.

[6] J. Lindenstrauss and L. Tzafriri, Classical Banach spaces II. Function spaces (Springer-Verlag, Berlin, 1979).

[7] H. H. Schaefer, Banach lattices and positive operators (Springer-Verlag, Berlin, 1974). 